\title{
DIRECT PRODUCT DECOMPOSITION OF ALTERNATIVE RINGS
}

\author{
HYO CHUL MYUNG AND LUIS R. JIMENEZ
}

\begin{abstract}
It is shown that any alternative ring $A$ equipped with the relation $\leq$, defined by $x \leq y$ if and only if $x y=x^{2}$, is isomorphic to a direct product of alternative division rings if and only if the relation $\leq$ is a partial order on $A$ such that $A$ is hyperatomic and orthogonally complete.
\end{abstract}

The result stated in the abstract was first proved by Abian [1] for the commutative associative case. Later, Chacron [4] extended this result to an arbitrary associative ring. While Chacron proved this by using the axiom of choice or a subdirect sum representation of any associative ring without nonzero nilpotent elements, Abian [2] recently gave another method to prove the result of Chacron without using the axiom of choice. It is the aim of this paper to extend the above result for the associative case to any altemative ring.

1. Preliminaries. An altemative ring is a nonassociative ring $A$ satisfying $x^{2} y=x(x y)$ and $y x^{2}=(y x) x$ for all $x, y \in A$. In terms of the associator $(x, y, z)=(x y) z-x(y z)$, this is to say $(x, x, y)=(y, x ; x)=0$. Thus an associative ring is altemative, and there exist altemative rings without nonzero nilpotent elements which are not associative. For this, see, for example, [3] or [6].

We recall some of the well-known properties in an altemative ring $A$ on which our proofs will be based. Artin's theorem in $A$ says that

any subring of $A$ generated by two el ements is associative.

Moufang identities are

$$
\begin{gathered}
(a x a) y=a[x(a y)], \\
a(x y) a=(a x)(y a) \text { for all } x, y \in A .
\end{gathered}
$$

Also, the following generalized version of Artin's theorem is proved in [3]:

Presented to the Society, September 24, 1973 under the title Direct product decomposition of alternative rings without nilpotent elements; received by the editors September 26, 1973.

AMS (MOS) subject classifications (1970). Primary 17D05.

Key words and phrases. Alternative ring, nilpotent element, direct product, hyperatom, orthogonal complete. 


$$
\text { if }(a, b, c)=0 \text {, the subring of } A \text { generated }
$$
by $a, b, c$ is associative.

If $A$ is an altemative ring without nonzero nilpotent elements, the following, in view of Artin's theorem, can be shown to be exactly the same as the associative case [2].

$$
\begin{gathered}
x^{2}=0 \text { for } x \in A \text { if and only if } x=0 . \\
x y=0 \text { for } x, y \in A \text { if and only if } y x=0 .
\end{gathered}
$$

For any elements $x, y, t \in A, x y=x t$

if and only if $y x=t x$.

Hence, in particular, if $x y=x^{2}$ then $x y=y x$.

2. Main section. In what follows, $A$ always stands for an alternative ring without nonzero nilpotent elements. First we prove a sequence of lemmas which are essential for the main result.

Lemma 1. Let $A$ be an altemative ring. The relation defined by $x \leq y$ if and only if $x y=x^{2}$ is a partial order on $A$ if and only if $A$ has no nonzero nilpotent elements.

Proof. Suppose $A$ has no nonzero nilpotent el ements. Then clearly $\leq$ is.reflexive. If $x \leq y$ and $y \leq x$, that is, $x y=x^{2}$ and $y x=y^{2}$, then we get $(x-y)^{2}=0$ and so $x=y$. To show transitivity of $\leq$ let $x \leq y$ and $y \leq z$. We first show

$$
x^{2} z x=x z x^{2}=x^{4} .
$$

Since $x y=x^{2}$ and $y z=y^{2}$, we have

$$
\begin{aligned}
x^{2} z x & =x^{2}(z x)=(x y)(z x)=x(y z) x \quad(\text { by }(3)) \\
& =x y^{2} x=(x y)^{2} \quad(\text { by }(7)) \\
& =x^{4} .
\end{aligned}
$$

Similarly, we show $x z x^{2}=x^{4}$. Using (8) we compute

$$
\left(x z x-x^{3}\right)^{2}=x z x^{2} z x-x z x^{4}-x^{4} z x+x^{6}=x^{4} z x-x^{6}-x^{4} z x+x^{6}=0
$$

and so

$$
x z x=x^{3} .
$$

Finally, from (8) and (9), we derive $\left(x z-x^{2}\right)^{2}=x^{3} z-x^{4}-x^{3} z+x^{4}=0$, and so $x z=x^{2}$ or $x \leq z$. Thus $\leq$ is transitive and is a partial order on $A$. 
The converse is easy and the same as in the associative case.

Henceforth any order in $A$ will mean the relation defined by $x \leq y$ if and only if $x y=x^{2}$. The following lemma is proved by Hentzel [5]. For convenience, we duplicate it here.

Lemma 2. Let $x, y, z$ be any elements of $A$. Then $(x y) z=0$ if and only if $x(y z)=0$.

Proof. By repeated use of (1) and (3), if $(x y) z=0$ then

$$
\begin{aligned}
(x \cdot y z)^{3} & =x[y z \cdot(x \cdot y z \cdot x) \cdot y z]=x[y z \cdot(x y \cdot z x) \cdot y z] \\
& =x[(y z \cdot x y)(z x \cdot y z)]=x[(y z \cdot x y)(z \cdot x y \cdot z)]=0
\end{aligned}
$$

and so $x(y z)=0$. The converse is similar.

Lemma 3. (i) For every $x, a \in A$, if $x^{2} a=0$ then $x a=0$.

(ii) If $x \leq y$ for $x, y \in A$, then $(x, y, A)=0$. Hence for every element $u \in A$, the subring of $A$ generated by $x, y, u$ is associative.

(iii) If $a b \leq c$ for $a, b, c \in A$, then the subring generated by $a, b, c$ is associative.

Proof. (i) In view of (1), the proof is the same as in the associative case.

(ii) Since $x y=x^{2}$,

$$
\begin{aligned}
0 & =\left(x y-x^{2}\right) u=[x(y-x)] u=x[(y-x) u] \quad(\text { Lemma 2) } \\
& =x(y u-x u)=x(y u)-x(x u)=x(y u)-x^{2} u .
\end{aligned}
$$

Hence $(x y) u=x^{2} u=x(y u)$ and $(x, y, u)=0$ for all $u \in A$, as desired.

(iii) In view of (4), it suffices to show $(a, b, c)=0$. Since $(a b) c=$ $(a b)^{2}$, using Lemma 2 we have

$$
0=(a b)(c-a b)=a \cdot b(c-a b)=a(b c-b a b)=a(b c)-(a b)^{2},
$$

and so $(a, b, c)=0$.

Lemma 4. For every element $x, y, u, v \in A, x \leq y$ and $u \leq v$ imply $x u \leq y v$.

Proof. We first show that for every $v \in A, x \leq y$ implies $x v \leq y v$ and $v x \leq v y$. Since $x y=x x$, by Lemma 3(ii), $(x y) v=x(y v)=x(x v)$. So by (7) we get $(y v) x=x v x$ and $y v x v=x v x v=(x v)^{2}$, so $x v \leq y v$; the other case is similar. Since $x u \leq y u$ and $y u \leq y v$, by transitivity (Lemma 1) we have that $x u \leq y v$. 
Lemma 5. Let $e$ be an idempotent in A. Then:

(i) $e x=x e \leq x$ for every $x \in A$.

(ii) $e$ is central in $A$; that is, $e$ commutes with every element in $A$ and $(e, A, A)=0$.

Proof. (i) is trivial. For (ii), one easily checks $(e x-e x e)^{2}=(x e-e x e)^{2}$ $=0$ and so $e x=x e$. Now, for every element $x, y \in A$,

$$
\begin{aligned}
0 & =[e(e x-x)](e y) \quad(\text { Lemma 2) } \\
& =e[(e x)(y e)-x(e y)] \quad(\text { Moufang) } \\
& =e[e(x y) e-x(e y)]=e(x y)-e[x(e y)] \quad \text { (Moufang) } \\
& =e(x y)-(e x e) y=e(x y)-(e x) y,
\end{aligned}
$$

as desired.

Lemma 6. Let $a, s$ be elements in $A$ such that $a^{2} s=a$. Then we have

(i) $a s a=a$;

(ii) as and sa are idempotents and as = sa;

(iii) if $x \leq$ as for $x \in A$, then $x$ is an idempotent;

(iv) $\left(a^{2}, s, A\right)=0$.

Proof. (i) One easily checks $(a s a-a)^{2}=0$.

(ii) Using (i), we get $(s a)^{2}=(s a)(s a)=s(a s a)=s a$, and similarly $(a s)^{2}=a s$. This then implies $(s a-a s)^{2}=0$ and so $s a=a s$.

(iii) If $x \leq a s$, then $x^{2} \leq x(a s)$ and $x(a s) \leq x$ by Lemma $5(i)$ since as is an idempotent. Hence $x^{2} \leq x$ by Lemma 1 ; that is, $x^{4}=x^{3}$. Using this, we get $\left(x^{3}-x^{2}\right)^{2}=0$ and so $x^{3}=x^{2}$, which means $x \leq x^{2}$ and so, by Lemma $1, x^{2}=x$.

(iv) Let $y$ be any element in $A$. From (2) and (6), we get $0=(a, a s, y)$ $=(a, s, y) a=a(a, s, y)$. Also, a known identity for $A$ says that $\left(a^{2}, s, y\right)$ $=a(a, s, y)+(a, s, y) a$ (see $[6, \mathrm{p} .129])$. Hence $\left(a^{2}, s, y\right)=0$, as desired.

Following Abian [1], we have

Definition. A nonzero element $a$ in $A$ is called a hyperatom in $A$ if and only if, for every element $x$ in $A$,

(i) $x \leq a$ implies $x=0$ or $x=a$, and

(ii) $a x \neq 0$ implies $a(x s)=a$ for some element $s$ in $A$.

We now prove the following crucial lemma.

Lemma 7. Let $x$ be a nonzero element in $A$. If $a \leq x$ for some nonzero hyperatom $a$ in $A$, then there exists an idempotent hyperatom $e$ such that $e x \neq 0$. 
Proof. Since $a$ is a hyperatom, by definition there exists an element $s \in A$ with $a^{2} s=a$. Then by Lemma 6(ii), $a s=s a$ is an idempotent. Hence it suffices to show as is a hyperatom and (as) $x \neq 0$. Let $y \leq a s$ for $y \in A$. Then $y^{2}=y(a s)$ and $y a \leq a s a=a$ by Lemmas 4 and $6(\mathrm{i})$. Thus $y a=0$ or $y a=a$. If $y a=0,0=(y a) s=y(a s)=y^{2}$ by Lemma 2 and so $y=0$. Suppose now $y a=a$. Then we get $(a, s, y)=0$ since $y$ is an idempotent by Lemma 6(iii). Thus $y=y^{2}=y(a s)=(y a) s=a s$. Hence $y=0$ or $y=a s$. Now suppose (as) $y \neq 0$ for $y \in A$. Then $a(s y) \neq 0$ by Lemma 2 and so $a^{2}(s y) \neq 0$, but then $a^{2}(s y)=\left(a^{2} s\right) y=a y \neq 0$ by Lemma $6(\mathrm{iv})$. Since $a$ is a hyperatom, there exists an $r \in A$ such that $a(y r)=a$, and hence $\left(a^{2} s\right)(y r)=a s a=a^{2} s s a$;

$$
0=\left(a^{2} s\right)(y r-s a)=a^{2}[s(y r-s a)] \quad(\text { Lemma } 2),
$$

and so

$$
0=a[s(y r-s a)]=(a s)(y r-s a) .
$$

Hence $(a s)(y r)=(a s)^{2}=a s$, and this proves as is a hyperatom. Finally if we let $e=a s$, then

$$
\begin{aligned}
e x & =(s a) x=s(a x) \quad(\text { by Lemma 3(ii)) } \\
& =s a^{2}=(s a) a=a s a=a \neq 0
\end{aligned}
$$

by Lemmas $5(\mathrm{ii})$ and $6(\mathrm{i})$.

Lemma 8. The set $E=\left\{e_{i}\right\}_{i \in I}$ of all idempotent hyperatoms in $A$ is an orthogonal set in $A$, and each $e_{i} A$ is an alternative division ring such that $e_{i} A \cap e_{j} A=0$ if $i \neq j$.

Since each $e_{i} A$ has no zero divisors, noting that each $e_{i}$ is central and that if every nonzero element is an alternative ring has a right inverse it has also a left inverse [6, p. 131], the proof is the same as in the associative case.

As in the associative case, we make the following

Definition. Let $A$ be an alternative ring.

(i) $A$ is called hyperatomic if for every nonzero element $r$ in $A$ there exists a hyperatom $a$ in $A$ such that $a \leq r$.

(ii) $A$ is called orthogonally complete if sup $S$ exists for every orthogonal subset $S$ of $A$.

The following lemma is an easy consequence of Lemma 7 (also see [1] and [4]).

Lemma 9. Let $A$ be hyperatomic and $E=\left\{e_{i}\right\}_{i \in I}$ be the set of idempotent hyperatoms in $A$. Then we have 
(i) for every $a \in A, \sup \left(e_{i} a\right)$ exists and $a=\sup \left(e_{i} a\right)$;

(ii) the function $f$ defined by $f(a)=\left(\dot{e}_{i} a\right)_{i \in I}$ is a monomorphism from $A$ into a direct product of the alternative division rings $e_{i} A$.

For the proof of the main result, we need one more lemma.

Lemma 10. Let $\left\{x_{i}\right\}_{i \in I}$ be a subset of the alternative ring $A$ such that $\sup _{i} x_{i}$ exists. Then for every element $a \in A, \sup _{i}\left(a x_{i}\right)$ exists and furthermore,

$$
a \sup _{i} x_{i}=\sup _{i}\left(a x_{i}\right)
$$

Proof. Let $u$ be any upper bound of $\left\{a x_{i}\right\}_{i \in I^{\circ}}$. We proceed as in [2]. Since $a x_{i} \leq u$,

$$
u\left(a x_{i}\right)=\left(a x_{i}\right)^{2} \text { for every } i \in I .
$$

By Lemma 4, $a$ sup $x_{i}$ is an upper bound of $\left\{a x_{i}\right\}_{i \in I}$ and so

$$
\left(a \sup _{i} x_{i}\right)\left(a x_{i}\right)=\left(a x_{i}\right)^{2} \text { for every } i \in I \text {. }
$$

From (10) and (11) we have

$$
u\left(a x_{i}\right)=\left(a \sup _{i} x_{i}\right)\left(a x_{i}\right) \text { for every } i \in I .
$$

Thus

$$
x_{i}^{2}=\left(\sup _{i} x_{i}\right) x_{i}+u\left(a x_{i}\right)-\left(a \sup _{i} x_{i}\right)\left(a x_{i}\right)
$$

Since $a x_{i} \leq u$ for every $i \in I$, from Lemma 3(iii) and 3(ii) applied to $x_{i}$, $\sup _{i} x_{i}, a$, we have

$$
\begin{aligned}
x_{i}^{2} & =\left(\sup _{i} x_{i}\right) x_{i}+(u a) x_{i}-\left[\left(a \sup _{i} x_{i}\right) a\right] x_{i} \\
& =\left[\sup _{i} x_{i}+u a-\left(a \sup _{i} x_{i}\right) a\right] x_{i}=x_{i}\left[\sup _{i} x_{i}+u a-\left(a \sup _{i} x_{i}\right) a\right] .
\end{aligned}
$$

Thus

$$
x_{i} \leq \sup _{i} x_{i}+u a-\left(a \sup _{i} x_{i}\right) a
$$

for every $i \in I$, which means

$$
\left(\sup _{i} x_{i}\right)^{2}=\left(\sup _{i} x_{i}\right)\left[\sup _{i} x_{i}+u a-\left(a \sup _{i} x_{i}\right) a\right] \text {, }
$$


which implies

$$
\left(\sup _{i} x_{i}\right)(u a)=\left(\sup _{i} x_{i}\right)\left(a \sup _{i} x_{i}\right) a \text {. }
$$

Setting $x=\sup _{i} x_{i}$, from (12) we get $x(u a)=x a x a$. Applying Lemma 2 to this,

$$
0=x(u a-a x a)=x \cdot(u-a x) a=x(u-a x) \cdot a=(x u) a-x a x a .
$$

Hence $(x u) a=x(u a)=(x a)^{2}$ and $a(x u)=(a x) u=(a x)^{2}$ or $a x \leq u$. Since $a x$ is an upper bound of $\left\{a x_{i}\right\}_{i \in I}$, this proves $a x=a \sup _{i} x_{i}=\sup _{i}\left(a x_{i}\right)$, as desired.

Remark. Lemmas 1, 4, and 10 generalize the results of Abian [2] to the altemative case. Also, our present results are extended to right altemative rings of characteristic $\neq 2$, since Kleinfeld [7] shows that right altemative rings of characteristic $\neq 2$ without nonzero nilpotent elements are alternative.

We are now prepared to prove the main result.

Theorem. Any alternative ring $A$ equipped with the relation $\leq$, defined by $x \leq y$ if and only if $x y=x^{2}$, is isomorphic to a direct product of alternative division rings if and only if the relation $\leq$ is a partial order on $A$ such that $A$ is hyperatomic and orthogonally complete.

The proof of the Theorem is a consequence of Lemmas 9, 8, and 10, and thus the same as in the associative case (see [1] and [4]).

The following example shows that Lemma 1 and the Theorem do not hold for Jordan rings.

Example. Let $Q$ be the real quaternions with the standard basis $1, i, j$, $k$. Then $Q$ under the product $a \cdot b=1 / 2(a b+b a)$ becomes a Jordan ring $Q^{+}$ without nonzero nilpotent elements. One easily checks in $Q^{+}$that $i+j \leq 2 i$ and $2 i \leq 2 i+j$ hold but $i+j \leq 2 i+j$ does not. Thus the relation $\leq$ is not a partial order on $Q^{+}$. Also, $Q^{+}$is a Jordan division ring in the sense that $U_{a}=2 R_{a}^{2}-R_{a^{2}}$ is invertible on $Q^{+}$for every $a \neq 0$ in $Q^{+}$, where $R_{a}$ is the right multiplication in $Q^{+}$by $a$.

\section{REFERENCES}

1. A. Abian, Direct product decomposition of commutative semisimple rings, Proc. Amer. Math. Soc. 24 (1970), 502-507. MR 41 \#3461.

2. A. Abian, Order relation in rings without nilpotent elements (preprint).

3. R. H. Bruck and E. Kleinfeld, The structure of alternative division rings, Proc. Amer. Math. Soc. 2 (1951), 878-890. MR 13, 526.

4. M. Chacron, Direct product of division rings and a paper of Abian, Proc. Amer. Math. Soc. 29 (1971), 259-262. MR 43 \#275.

5. I. R. Hentzel, Alternative rings without nilpotent elements, Proc. Amer. Math. Soc. 42 (1974), 373-376. 
6. E. Kleinfeld, A characterization of the Cayley number, Studies in Modern Algebra, edited by A. A. Albert, Math. Assoc. Amer., 1963, pp. 126-143.

7. - Right alternative rings, Proc. Amer. Math. Soc. 4 (1953), 939-944. MR 15, 595 .

DEPARTMENT OF MATHEMATICS, UNIVERSITY OF NORTHERN IOWA, CEDAR IOWA 50613 (Current address of H. C. Myung)

Current address (Luis R. Jimenez): Department of Mathematics, Universidad Pedagogica Nacional, Bogotá, Colombia, South America 